pressure at different temperature levels. An attempt has been made to develop crack-stopping mechanisms that are easy to install in existing pipelines; nevertheless, to achieve an optimum installation, these mechanisms may require further improvements.

\section{REFERENCES}

1. 'ISO TC 138: CD 13'477, Resistance to crack propagation: Determination of the critical pressure for rapid crack propagation (Small Scale Steady State [S4])' (ISO).

2. Yayla, P., 'Rapid crack propagation in polyethylene gas pipes', Ph.D. Thesis, Department of Mechanical Engineering, Imperial College, London, January, 1991.

3. Leevers, P. S., Venizelos, G. and Ivankovic, A., 'Rapid crack propagation along pressurised PE pipe: small-scale testing and numerical modelling', 'Proc. Plastics Pipes VIII', (The Plastics and Rubber Institute, The Netherlands, 1992) pp. D1/7-1-11.
4. 'ISO/TR 9080: Methods of extrapolation of hydrostatic rupture data to determinate long-term hydrostatic strength of thermoplastic pipe materials' (ISO).

5. Farshad, M. and Flüeler, P., 'Crack propagation in cylindrical shells and pipes', Int. J. Engng Fracture Mech. 44 (1993) 937-947

6. Flüeler, P. and M. Farshad, M., 'Schnelle Rissausbreitungeine Besonderheit im Bruchverhalten von KunststoffRohren', gwa (Gas, Wasser, Abwasser, Schweizerischen Vereins des Gas-und Wasserfaches), 72 Jahrgang (1992) pp. $350-352$.

7. Farshad, $M$. and Flüeler, $P$., 'Crack propagation in prestressed plates', Int. J. Engng Fracture Mech. (1995) in press.

8. Idem, 'Dynamic fracture toughness of thermoplastic pipes rapid crack propagation in PE-HD pipes', EMPA internal Report No. 150'317/1, September 1994.

\title{
Fibre optic sensors for civil structures monitoring
}

\author{
U. SENNHAUSER, R. BRÖNNIMANN, PH. M. NELLEN \\ EMPA Department Electronics/Metrology
}

\section{INTRODUCTION}

In recent years an increasing quantity of advanced composites and other new materials as well as construction methods have been applied in buildings, prestressed tendons, bridges, etc. Without long term experience of their ageing behaviour it is important for us to apply efficient tools for monitoring their quality and long term stability.

Sensors used today broadly for this purpose are, e.g., electrical resistance strain gauges, displacement transducers, extensometers and optical systems for special applications. In academic research much attention has been given to the very attractive idea of using optical fibres for sensing all kinds of physical, chemical and biological effects. Techniques using back or forward scattered light, polarization states or intensities in multi or mono mode fibres have been investigated, although few are competitive with conventional methods.

Of particular interest in civil engineering are fibre optic sensors for measuring strain and displacements and for detecting cracks $[1,2]$. Before they will become commercially acceptable, they have to be made more efficient, reliable, accurate, cheaper and easier to apply than the well established methods mentioned above.

In this work, low coherence interferometry is used for absolute strain measurements using embedded fibre Fabry-Perot sensors and fibre gratings with broad bandwidths. It matches the range and resolution of electrical resistance strain gauges, and its multiplexing capabilities make it a technically and economically promising tool for distributed sensing.

\section{FIBRE OPTIC SENSING SYSTEM}

Low coherence or white light interferometry attracts increasing attention for measuring absolute distances with interferometric resolutions of a few nanometres (nm). In long term field monitoring it is mandatory to use such an absolute method to avoid interrupt free measurements as required in normal laser interferometry and in measuring local strain distributions, e.g., $1 \mu \mathrm{m} \mathrm{m}^{-1}$ with a local resolution of $1 \mathrm{~cm}$, requires the measurement of relative displacements of $10 \mathrm{~nm}$.

A schematic of an experimental set-up is shown in Fig. 1. A broadband superluminescent light diode (SLD) has a sufficiently short coherence length of $26 \mu \mathrm{m}$ for unambiguous identification of the zero fringe and discrimination between different length sensing elements $\left(\mathrm{d}_{\mathrm{FP} 1 \ldots 3}\right)$ in the fibre in a parallel or serial multiplexing scheme. Low coherence demodulation is performed with a scanning Michelson interferometer with a moveable mirror $\mathrm{M}_{2}$ whose position is controlled permanently by

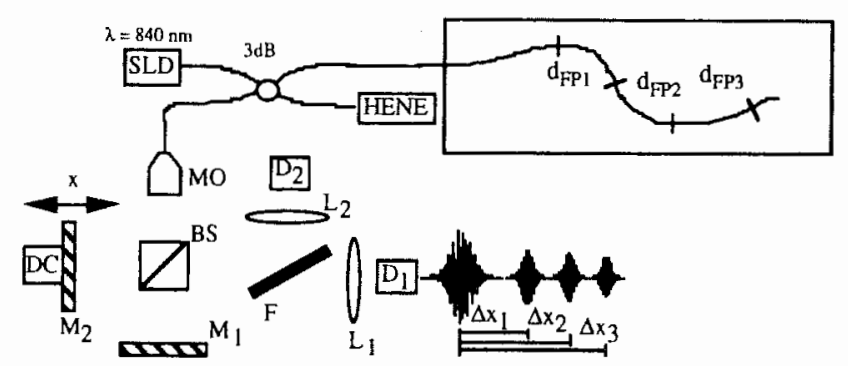

Fig. 1 Schematic drawing of the scanning Michelson interferometer with serially embedded FP sensor elements. MO, microscope objective; BS, beamsplitter; DC, DC motor; $M_{1,2}$, mirrors; $D_{1,2}$, detectors; $L_{1,2}$, lenses; $F$, filter. 

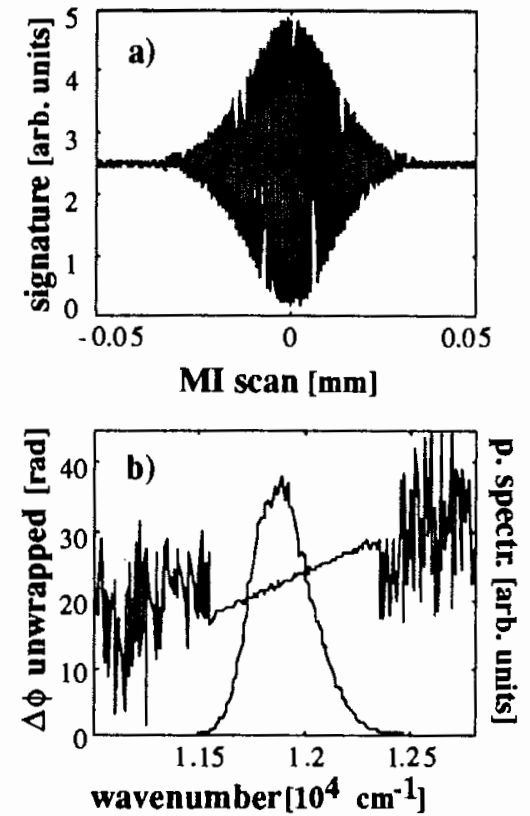

Fig. 2 (a) Main interference signature and (b) power spectrum of an FP signature with unwrapped phase difference.

a helium-neon laser. The length of a sensing element is then given by the travel distance $\Delta x$ of mirror $M_{2}$ between the main and a side signature.

The position of the maximum of the signature (Fig. 2a) is determined to sub-wavelength resolution by fast Fourier transformation (FFT) providing power spectra and corresponding unwrapped phase shift between light source and reflected light (Fig. 2b). Assuming a constant group index of refraction $N$ the local strain $\varepsilon_{\mathrm{FP}}$ of a straight fibre element subjected to uniaxial tension is given by [3]

$\varepsilon_{\mathrm{FP}}=\Delta\left(N d_{\mathrm{FP}}\right) /\left(A N d_{\mathrm{FP}}\right), A=1-N^{2} / 2\left(p_{12}-v\left(p_{11}+p_{12}\right)\right)$ $A=0.796$ includes change of geometric length (first term) and change of refractive index (second term), $p_{11}=0.113$ and $p_{12}=0.252$ [4] are the strain optic coefficients and $v=0.16$ is the Poisson ratio of the fibre material (fused silica). More details of data analysis have been given elsewhere [5]

\section{RESULTS AND DISCUSSION}

The strain of an aluminium cantilever beam has been measured simultaneously by a fibre optic Fabry-Perot (FP) interferometer of about $10 \mathrm{~mm}$ length and an electrical resistance strain gauge (RSG) of similar length. The two sensor types were glued to the beam with an epoxy adhesive in a configuration exposing them to the same strain both in compression and expansion. Fig. 3a shows low coherence demodulated FP measurements in comparison with RSG, and Fig. 3b shows the same with two FP sensors multiplexed in parallel. They show good linear behaviour over the investigated range of $500 \mu \mathrm{m} \mathrm{m}^{-1}$ with a slope of $\Delta \varepsilon_{\mathrm{FP}} / \Delta \varepsilon_{\mathrm{RSG}}=0.98 \pm 0.04$, which is consistent with unity. The resolution of $1-2 \mu \mathrm{m} \mathrm{m}^{-1}$ corresponds to a displacement measurement of $10-20 \mathrm{~nm}$ over the $10 \mathrm{~mm}$ long FP interferometers. The data had to be corrected for a temperature drift of the FP's of about $100 \mathrm{~nm} \mathrm{~K}-1$.
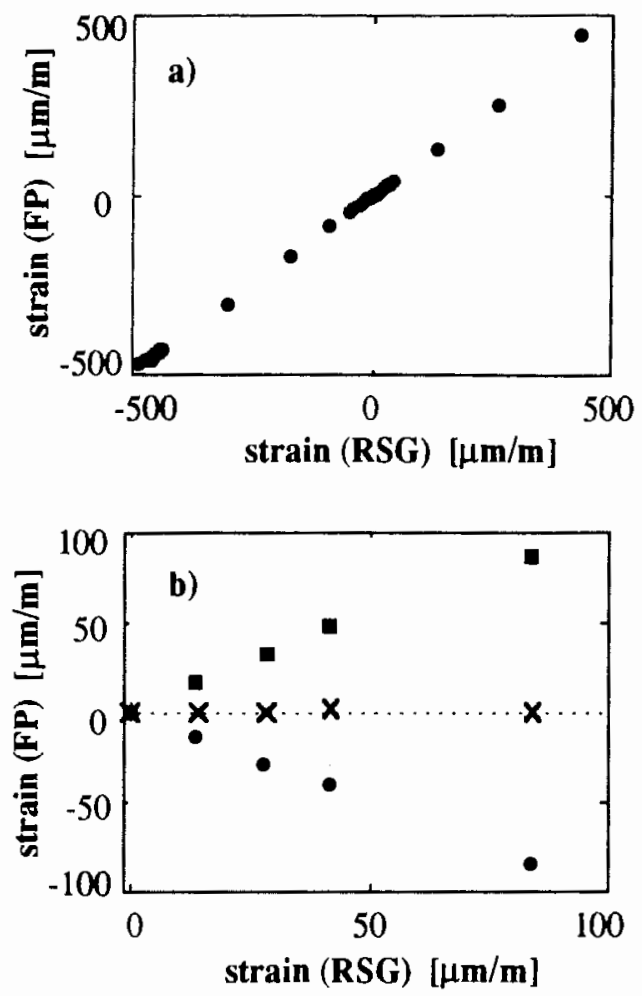

Fig. 3 Strain measurements with a single FP and a parallel FP configuration compared with RSG measurements in tension and compression.

\section{CONCLUSION}

Low coherence phase demodulated measurements with fibre optic Fabry-Perot interferometers have been demonstrated to give strain resolutions of $1 \mu \mathrm{m} \mathrm{m}^{-1}$ with a linear response. The experiments performed with a cantilever beam show excellent agreement with electrical resistance strain gauges over the tested range of $500 \mu \mathrm{m} \mathrm{m}^{-1}$. In order to get a competitive or, for some applications, even a superior instrument for strain measurements in civil structures, embedding and long term stability must be demonstrated and efficiency of application and data analysis must be improved.

\section{REFERENCES}

1. Measures, R. M., Alavie, T., Maaskant, R., Ohn, M., Karr, S., Glennie, D., Wade, C., Guha-Thakurta, A., Tadros, G. and Rizkalla, S., 'Multiplexed Bragg grating laser sensors for civil engineering', SPIE 2071 (1993) 21-29.

2. de Vries, M., Claus, R. O., Masri, S. and Mustafa, M., 'Application of embedded optical fibre sensors to monitoring of fatigue loading of reinforced concrete cross-beams', in Proceedings of OFS-9, 1993, pp. 389-392.

3. Sirkis, J. S., 'Unified approach to phase-strain-temperature models for smart structure interferometric optical fibre sensors development/application', Optical Engng. 32 (1993) 752-761; 762-772.

4. Bertholds, A. and Dändliker, R., 'Determination of the individual strain-optic coefficients in single-mode optical fibres, J. Lightwave Technol. 6 (1988) 17-20.

5. Nellen, Ph. M., Pierhöfer, H., Brönnimann, R. and Sennhauser, U., 'Absolute strain measurements with multiplexed low coherence demodulated fibre Fabry-Perot sensors', SPIE 2360 (1994) 518-520. 